\title{
A JORDAN CURVE SPANNED BY A COMPLETE MINIMAL SURFACE
}

\author{
FRANCISCO MARTÍN AND NIKOLAI NADIRASHVILI
}

\begin{abstract}
In this paper we construct complete (conformal) minimal immersions $f: \mathbb{D} \longrightarrow \mathbb{R}^{3}$ admitting continuous extensions to the closed disk, $F: \overline{\mathbb{D}} \longrightarrow \mathbb{R}^{3}$. Moreover, $F_{\mid \mathbb{S}^{1}}: \mathbb{S}^{1} \rightarrow F\left(\mathbb{S}^{1}\right)$ is a homeomorphism and $F\left(\mathbb{S}^{1}\right)$ is a (non-rectifiable) Jordan curve with Hausdorff dimension 1.

It turns out that the set of Jordan curves $F\left(\mathbb{S}^{1}\right)$ constructed by the above procedure is dense in the space of Jordan curves of $\mathbb{R}^{3}$ with the Hausdorff metric.
\end{abstract}

2000 Mathematics Subject Classification. Primary 53A10; Secondary 49Q05, 49Q10, 53C42. Key words and phrases: Complete minimal surfaces, Plateau problem.

\section{INTRODUCTION AND BACKGROUND}

The Belgian physicist J. Plateau carried out extensive experiments with soap films in the mid-nineteenth century. Out of his investigations he developed the mathematically important conjecture that every closed boundary curve that neither touches itself nor intersects itself can be spanned by a minimal surface. The problem of finding this surface for a given boundary curve entered mathematical history as the "Plateau problem".

A complete mathematical proof of its solvability was furnished first in 1931 by J. Douglas and T. Radó, independently of one another. Their result marked a brilliant high point in the calculus of variations. As has happened so frequently in the history of Mathematics, the solution of this long open problem immediately generated new questions. The theorem proved by Douglas and Radó stated only the mathematical existence of an area-minimizing surface for a given rectifiable curve, but said very little about its geometrical properties. Further development concentrated therefore on a better understanding of this solution of the Plateau problem, in particular its behavior close to the boundary curve. In particular, it was shown that any Jordan curve can be spanned by a minimal surface, [8]. We refer to [3] for more details.

In the last forty years, interest has concentrated on the global theory of complete minimal surfaces. This class of minimal surfaces are (apparently) far away from the original meaning of the term minimal surfaces (as solutions of Plateau problem): they are infinitely extended in the intrinsic distance. Because there were no sufficiently complicated examples for exact investigation, this new development proceeded only slowly. However, last few years have seen an important progress on many long-standing problems in global theory of complete minimal surfaces in $\mathbb{R}^{3}$. One of these has been the Calabi-Yau problem, which dates back to the 1960s. Calabi asked whether or not it is possible for a complete minimal surface in $\mathbb{R}^{3}$ to be contained in the ball $\mathbb{B}=\left\{x \in \mathbb{R}^{3} \mid\|x\|<1\right\}$. Much work has been done on it over the past four decades. The most important result in this line was obtained by the second author in [7] where he constructed a complete minimal surface in $\mathbb{B}$.

The study of the Calabi-Yau problem have generated new lines of work and techniques. Among other things, these new ideas have established a surprising relationship between the theory of complete minimal surfaces in $\mathbb{R}^{3}$ and the Plateau problem. In this sense, the main goal of this paper consists of finding Jordan curves in Euclidean space spanning complete minimal surfaces. One can think that such curves are rare, however we prove that Jordan curves of this kind are dense in the space of Jordan curves with the Hausdorff metric.

Theorem. There exist complete, conformal, minimal immersions $f: \mathbb{D} \longrightarrow \mathbb{R}^{3}$ so that they admit a continuous extension to the closed disk, $F: \overline{\mathbb{D}} \longrightarrow \mathbb{R}^{3}$. The map $F_{\mid \mathbb{S}^{1}}$ is an embedding and $F\left(\mathbb{S}^{1}\right)$ is a non-rectifiable Jordan curve with Hausdorff dimension 1.

Moreover, for any Jordan curve $\Gamma$ in $\mathbb{R}^{3}$ and any $\xi>0$, we can find a minimal immersion satisfying the above conditions and such that $\delta^{H}\left(\Gamma, F\left(\mathbb{S}^{1}\right)\right)<\xi$, where $\delta^{H}$ means the Hausdorff distance.

Date: February 10, 2006.

First author's research is partially supported by MEC-FEDER Grant no. MTM2004 - 00160. 
It is important to notice that $f$ cannot be an embedding as follows from the recent result by T. Colding and W. P. Minicozzi that asserts any complete embedded minimal surface in $\mathbb{R}^{3}$ with finite topology is proper in $\mathbb{R}^{3}$. In particular, such a surface cannot span a Jordan curve.

The proof of the main theorem requires several previous lemmas. Two of them (Lemmas 1 and 2) are related to the question: when is the limit of Jordan curves a Jordan curve ? This question is important for us because we obtain our complete minimal immersion as a limit of minimal disks with arbitrarily large intrinsic radius and bounded by Jordan curves. In order to construct this family of disks we need an approximation lemma (Lemma 4) which represents an important innovation in the construction of minimal surfaces related with the Calabi-Yau problem.

Lemma. Let $X: \overline{\mathbb{D}} \rightarrow \mathbb{R}^{3}$ be a conformal minimal immersion, so that $X\left(\mathbb{S}^{1}\right)$ is a Jordan curve. Then, for any $\mu>0$ there exists a conformal minimal immersion $Y: \overline{\mathbb{D}} \rightarrow \mathbb{R}^{3}$ satisfying:

(i) $\|Y-X\|<\mu$, in $\mathbb{D}$;

(ii) $\operatorname{dist}_{Y}\left(0, \mathbb{S}^{1}\right)>\operatorname{dist}_{X}\left(0, \mathbb{S}^{1}\right)+1$;

(iii) length $\left(Y\left(\mathbb{S}^{1}\right)\right)>$ length $\left(X\left(\mathbb{S}^{1}\right)\right)$;

(iv) $Y\left(\mathbb{S}^{1}\right)$ is a Jordan curve.

This lemma represents a powerful instrument that would allow, among other things, to simplify considerably the writing of most of the papers related with the existence of complete bounded minimal surfaces in $\mathbb{R}^{3}$.

The authors would like to thanks L. Ferrer for her careful reading of a preliminary version of this paper.

1.1. Minimal surface background. Given $X=\left(X_{1}, X_{2}, X_{3}\right): M \longrightarrow \mathbb{R}^{3}$ a conformal minimal immersion we denote by $g: M \longrightarrow \overline{\mathbb{C}}=\mathbb{C} \cup\{\infty\}$ its stereographically projected Gauss map that is a meromorphic function and by $\Phi_{3}$ the holomorphic differential defined as $\phi_{3}=d X_{3}+\star \mathrm{i} d X_{3}$, where $\star$ denotes the Hodge operator on $M$. The pair $\left(g, \Phi_{3}\right)$ is usually referred to as the Weierstrass data of the minimal surface, and the minimal immersion $X$ can be expressed, up to translations, solely in terms of these data as

$$
X=\operatorname{Re} \int^{z}\left(\Phi_{1}, \Phi_{2}, \Phi_{3}\right)=\operatorname{Re} \int^{z}\left(\frac{1}{2}\left(\frac{1}{g}-g\right), \frac{\mathrm{i}}{2}\left(\frac{1}{g}+g\right), 1\right) \Phi_{3},
$$

where Re stands for real part and $z$ is a conformal parameter on $M$. The pair $\left(g, \Phi_{3}\right)$ satisfies certain compatibility conditions:

$$
\begin{gathered}
\Phi_{1}^{2}+\Phi_{2}^{2}+\Phi_{3}^{2}=0 ; \\
\left\|\Phi_{1}\right\|^{2}+\left\|\Phi_{2}\right\|^{2}+\left\|\Phi_{3}\right\|^{2} \neq 0
\end{gathered}
$$

and all periods of the $\Phi_{j}$ are purely imaginary; $j=1,2,3$.

Conversely, if $M$ is a Riemann surface, $g: M \rightarrow \overline{\mathbb{C}}$ is a meromorphic function and $\Phi_{3}$ is a holomorphic oneform on $M$ fulfilling the conditions (2) and (3) then the map $X: M \rightarrow \mathbb{R}^{3}$ given by (1) is a conformal minimal immersion with Weierstrass data $\left(g, \Phi_{3}\right)$.

Condition ii) stated above deals with the independence of (1) on the integration path, and it is usually called the period problem. In this article, all the minimal immersions are defined on simply connected domains of $\mathbb{C}$. Then, the Weierstrass 1-forms have no periods, and so the only requirements are (2) and (3). In this case, the differential $\eta \stackrel{\text { def }}{=} \Phi_{3} / g$ can be written as $\eta=f(z) d z$. The metric of $X$ can be expressed as

$$
\mathcal{S}_{X}^{2}=\frac{1}{2}\|\Phi\|^{2}=\left(\frac{1}{2}\left(1+|g|^{2}\right)|f||d z|\right)^{2} .
$$

Throughout the paper, we will use several orthonormal bases of $\mathbb{R}^{3}$. Given $S$ an orthonormal basis and $v \in \mathbb{R}^{3}$, let $v_{(k, S)}$ denote the $k$-th coordinate of $v$ in $S$. The first two coordinates of $v$ in this basis will be represented by $v_{(*, S)}=\left(v_{(1, S)}, v_{(2, S)}\right)$.

Given a curve $\alpha$ in $M$, by length $(\alpha, X)$ we mean the length of $\alpha$ with respect to the metric $\mathcal{S}_{X}$. Given a subset $W \subset M$, we define:

- $\operatorname{dist}_{(W, X)}(p, q)=\inf \left\{\right.$ length $\left.\left(\alpha, \mathcal{S}_{X}\right) \mid \alpha:[0,1] \rightarrow W, \alpha(0)=p, \alpha(1)=q\right\}$, for any $p, q \in W$;

- $\operatorname{dist}_{(W, X)}\left(T_{1}, T_{2}\right)=\inf \left\{\operatorname{dist}_{\left(W, \mathcal{S}_{X}\right)}(p, q) \mid p \in T_{1}, q \in T_{2}\right\}$, for any $T_{1}, T_{2} \subset W$; 
The Euclidean metric on $\mathbb{C}$ will be denoted as $\langle\cdot, \cdot\rangle$. Note that $\mathcal{S}_{X}{ }^{2}=\lambda_{X}^{2}\langle\cdot, \cdot\rangle$, where the conformal coefficient $\lambda_{X}$ is given by (4).

Given a domain $D \subset \mathbb{C}$, we will say that a function, or a 1-form, is harmonic, holomorphic, meromorphic,... on $\bar{D}$, if it is harmonic, holomorphic, meromorphic,... on a domain containing $\bar{D}$. Most of the domains appearing in the paper will be disks. Given $r>0$, we define

$$
\mathbb{D}_{r}:=\{z \in \mathbb{C}|| z \mid<r\}, \quad \mathbb{S}_{r}^{1}:=\partial \mathbb{D}_{r}=\{z \in \mathbb{C}|| z \mid=r\} .
$$

For the sake of simplicity we will write $\mathbb{D}$ instead of $\mathbb{D}_{1}$ and $\mathbb{S}^{1}$ instead of $\mathbb{S}^{1}$.

\section{Preliminary Lemmas}

2.1. Some results on Jordan curves. Let us define $\mathcal{J}:=\left\{f: \mathbb{S}^{1} \longrightarrow \mathbb{R}^{3}\right.$ continuous embeddings $\}$ and $\mathcal{J}_{0}:=$ $\left\{f \in \mathcal{J}: f \in \mathcal{C}^{\infty}\left(\mathbb{S}^{1}, \mathbb{R}^{3}\right)\right\}$. Over this spaces of functions we are going to define the following operators:

$$
\begin{gathered}
\psi: \mathcal{J} \times \mathbb{N} \longrightarrow \mathbb{R}^{+} \\
\psi(f, n):=\frac{\inf \left\{\left\|f\left(x_{1}\right)-f\left(x_{2}\right)\right\|:\left|x_{1}-x_{2}\right|>1 / n\right\}}{n^{2}},
\end{gathered}
$$

and

$$
\begin{gathered}
\varphi: \mathcal{J}_{0} \times \mathbb{N} \longrightarrow \mathbb{R}^{+} \\
\varphi(f, n):=\min \left\{\psi(f, n), \frac{1}{n^{2}(\operatorname{length}(f))^{n}}\right\} .
\end{gathered}
$$

With this notation, the next two lemmas can be stated:

Lemma 1. Let $\left\{f_{n}\right\}_{n \in \mathbb{N}}$ be a sequence in $\mathcal{J}$ satisfying:

$$
\left\|f_{n}-f_{n-1}\right\|<\psi\left(f_{n-1}, n\right), \quad \forall n \in \mathbb{N}
$$

then $\left\{f_{n}\right\}_{n \in \mathbb{N}}$ converges uniformly to an element $f \in \mathcal{J}$.

Proof. From our assumptions, the sequence $\left\{f_{n}\right\}_{n \in \mathbb{N}}$ converges uniformly to a continuous map $f: \mathbb{S}^{1} \rightarrow \mathbb{R}^{3}$. We only need to check that $f$ is one-to-one.

Consider $\zeta_{1}$ and $\zeta_{2}$ two different points in $\mathbb{S}^{1}$ and label $a=\left|\zeta_{1}-\zeta_{2}\right|>0$. Take $n \in \mathbb{N}$ so that $n>1 / a$. Then, it is straightforward to see that:

$$
\left\|f_{n+k}\left(\zeta_{1}\right)-f_{n+k}\left(\zeta_{2}\right)\right\|>\left\|f_{n}\left(\zeta_{1}\right)-f_{n}\left(\zeta_{2}\right)\right\| \prod_{m=n}^{k}\left(1-\frac{1}{m^{2}}\right) .
$$

If we take limits in (5) as $k \rightarrow \infty$, then we obtain that $\left\|f\left(\zeta_{1}\right)-f\left(\zeta_{2}\right)\right\| \geq \frac{1}{2}\left\|f_{n}\left(\zeta_{1}\right)-f_{n}\left(\zeta_{2}\right)\right\|>0$. This completes the proof.

Lemma 2. Let $\left\{f_{n}\right\}_{n \in \mathbb{N}}$ be a sequence in $\mathcal{J}_{0}$ such that:

(i) $\left\|f_{n}-f_{n-1}\right\|<\varphi\left(f_{n-1}, n\right), \quad \forall n \in \mathbb{N}$,

(ii) length $\left(f_{n}\right)>\operatorname{length}\left(f_{n-1}\right), \quad \forall n \in \mathbb{N}$,

(iii) $\lim _{n \rightarrow \infty} \operatorname{length}\left(f_{n}\right)=+\infty$.

Then the Hausdorff dimension of $f\left(\mathbb{S}^{1}\right)$ is 1 , where $f=\lim _{n} f_{n}$.

Proof. First of all, notice that Lemma 1 implies that the sequence $\left\{f_{n}\right\}_{n \in \mathbb{N}}$ converges to an element $f \in \mathcal{J}$.

Fix $n \in \mathbb{N}$ so that $\sum_{k=n}^{\infty} \frac{1}{k^{2}}<1$, and length $\left(f_{k}\right)>1$, for all $k \geq n$. We are going to prove that:

$$
\left\|f_{k}-f_{n}\right\|<\frac{1}{\operatorname{length}\left(f_{n}\right)^{n}}, \quad \text { for all } k>n \text {. }
$$

Indeed, notice that we can successively apply hypothesis (i) in the lemma to deduce:

$$
\left\|f_{k}-f_{n}\right\| \leq \frac{1}{k^{2} \text { length }\left(f_{k-1}\right)^{k}}+\frac{1}{(k-1)^{2} \operatorname{length}\left(f_{k-2}\right)^{k-1}}+\cdots+\frac{1}{(n+1)^{2} \text { length }\left(f_{n}\right)^{n+1}},
$$




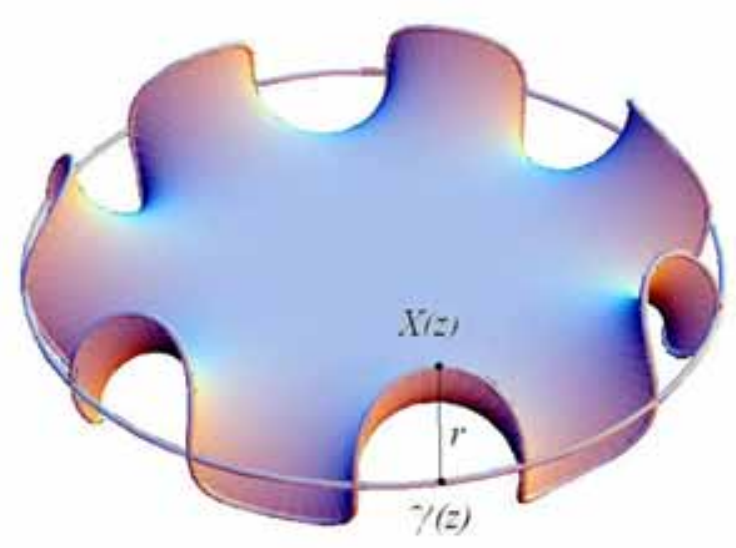

FIGURE 1. The minimal disk $X(\mathbb{D})$ and the Jordan curve $\Gamma$.

now we use hypothesis (ii) to obtain

$$
\left\|f_{k}-f_{n}\right\|<\frac{1}{\operatorname{length}\left(f_{n}\right)^{n}}\left(\sum_{k=n+1}^{\infty} \frac{1}{k^{2}}\right)<\frac{1}{\operatorname{length}\left(f_{n}\right)^{n}}
$$

If we take limit in (6) as $k$ goes to infinity, then we have

$$
\left\|f-f_{n}\right\|<\frac{1}{\operatorname{length}\left(f_{n}\right)^{n}}, \quad \text { for any } n \text { sufficiently large. }
$$

From now on we denote $s=1 / \operatorname{length}\left(f_{n}\right)^{n}$ and $N=\mathrm{I}\left(\right.$ length $\left.\left(f_{n}\right) / s\right)$, where $\mathrm{I}(x)$ means the integer part of $x$. Let $a_{1}, \ldots, a_{N}$ be the points on $f_{n}\left(\mathbb{S}^{1}\right)$ with mutual distance $s$ along $f_{n}\left(\mathbb{S}^{1}\right)$. Consider $B_{i}$ the ball centered at $a_{i}$ of radius $2 s, i=1, \ldots, N$. Then the union of all $B_{i}$ covers the curve $f\left(\mathbb{S}^{1}\right)$. Moreover, it is clear that $N(2 s)^{1+1 / n}<4$. This particularly means that the Hausdorff measure $\mathcal{H}^{1}\left(f\left(\mathbb{S}^{1}\right)\right)<\infty$ and so the Hausdorff dimension $\operatorname{dim}\left(f\left(\mathbb{S}^{1}\right)\right)$ is at most 1 (we refer to [6] for more details about Hausdorff measure.) But the Hausdorff dimension of $f\left(\mathbb{S}^{1}\right)$ is at least 1 (it is a Jordan curve.) This completes the proof.

2.2. The approximation lemmas. Lemmas in this section represent the kernel of this paper. In particular Lemma 4 is the most important one. In order to prove Lemma 4 we need first an intermediate result (Lemma 3) that concentrates all the technical computations required in the main lemma. Although the principal ideas in the proof of Lemma 3 are new, it is inspired in a similar result proved by the first author and S. Morales in [5, Lemma 1].

Lemma 3 (Completeness Lemma). Consider $\Gamma$ a closed analytic curve and let $\gamma: \mathbb{S}^{1} \rightarrow \Gamma$ be a parametrization of $\Gamma$. Let be $X: \overline{\mathbb{D}} \rightarrow \mathbb{R}^{3}$ a conformal minimal immersion, and $r, \rho$, positive constants satisfying:

(i) $\operatorname{dist}_{(\overline{\mathbb{D}}, X)}\left(0, \mathbb{S}^{1}\right)>\rho$;

(ii) $X\left(\mathbb{S}^{1}\right)$ is a Jordan curve;

(iii) $\|X(z)-\gamma(z)\|<r$, for all $z \in \mathbb{S}^{1}$.

Then, for all $\epsilon, s>0$ there exists a conformal minimal immersion $\widetilde{X}: \overline{\mathbb{D}} \rightarrow \mathbb{R}^{3}$ verifying:

(a) $\left.\operatorname{dist}_{(\bar{D}}, \tilde{X}\right)\left(0, \mathbb{S}^{1}\right)>\rho+s$;

(b) $\tilde{X}\left(\mathbb{S}^{1}\right)$ is a Jordan curve;

(c) $\|\tilde{X}(z)-\gamma(z)\|<R$, for all $z \in \mathbb{S}^{1}$, where $R=\sqrt{(2 s)^{2}+r^{2}}+\epsilon$.

Proof. Fix a positive integer $N>3$, satisfying the following:

- $2 / N<\epsilon$;

- $\operatorname{dist}_{(\overline{\mathbb{D}}, X)}\left(0, \mathbb{S}^{1}{ }_{1-2 / N}\right)>\rho$;

- $\|X(z)-\gamma(z /|z|)\|<r$, for all $z \in \overline{\mathbb{D}}-\mathbb{D}_{1-2 / N}$. 
Notice that the last inequality directly follows from hypothesis (ii), provided that $N$ is sufficiently large.

The first step will consist of describing a labyrinth on $\overline{\mathbb{D}}-\mathbb{D}_{1-2 / N}$, which depends on $N$.

Remark 1. Throughout the proof of the lemma a set of real positive constants depending on $X, r, \epsilon$ and $s$ will appear. These constants will be represented by the symbol 'const.'. Notice that the choice of these constants does not depend on the integer $N$.

Consider $n \in\left\{1, \ldots, 2 N^{2}\right\}, m \in\{1, \ldots, N\}$ and label $s_{n}=1-n / N^{3}$. We define the following subsets of $\mathbb{D}$ (see Fig. 2):

- If $n$ is odd:

$$
\Omega_{N}^{m, n}=\left\{z \in \mathbb{C}: s_{n}+\frac{1}{4 N^{3}} \leq|z| \leq s_{n-1}-\frac{1}{4 N^{3}} \quad\right. \text { and }
$$

$$
\left.2(m-1) \frac{\pi}{N}+\frac{1}{N^{2}} \leq \arg (z) \leq 2 m \frac{\pi}{N}-\frac{1}{N^{2}}\right\}
$$

- If $n$ is even:

$$
\begin{aligned}
\Omega_{N}^{m, n}=\left\{z \in \mathbb{C}: s_{n}+\frac{1}{4 N^{3}} \leq|z| \leq s_{n-1}-\frac{1}{4 N^{3}}\right. & \text { and } \\
& \left.2(m-1) \frac{\pi}{N}+\frac{1}{N^{2}} \leq \arg \left(z e^{i \frac{\pi}{N}}\right) \leq 2 m \frac{\pi}{N}-\frac{1}{N^{2}}\right\},
\end{aligned}
$$

We also define $\Omega_{N}$ as $\Omega_{N}=\bigcup_{m, n} \Omega_{N}^{m, n}$. Finally, let $L_{k}$ be the segment $L_{k}=\left\{z \in \mathbb{C}: \arg (z)=\frac{k \pi}{N}\right.$ and $s_{2 N^{2}}<$ $|z|<1\}$ for $k=1, \ldots, 2 N$. Let $\omega_{k}$ and $\varpi_{k}$ define:

$$
\begin{gathered}
\omega_{k}=L_{k} \cup\left(\bigcup_{(m, n) \in \Xi} \Omega_{N}^{m, n}\right), \quad \text { where } \Xi=\left\{(m, n): \Omega_{N}^{m, n} \cap L_{k} \neq \emptyset\right\}, \\
\varpi_{k}=\left\{z \in \mathbb{C}: \operatorname{dist}_{(\mathbb{C},<\cdot, \cdot>)}\left(z, \omega_{k}\right)<t\right\},
\end{gathered}
$$

being $1 / N>t>0$ sufficiently small so that $\bar{\varpi}_{1}, \ldots, \bar{\varpi}_{2 N}$ are pairwise disjoint (see Fig. 3.)

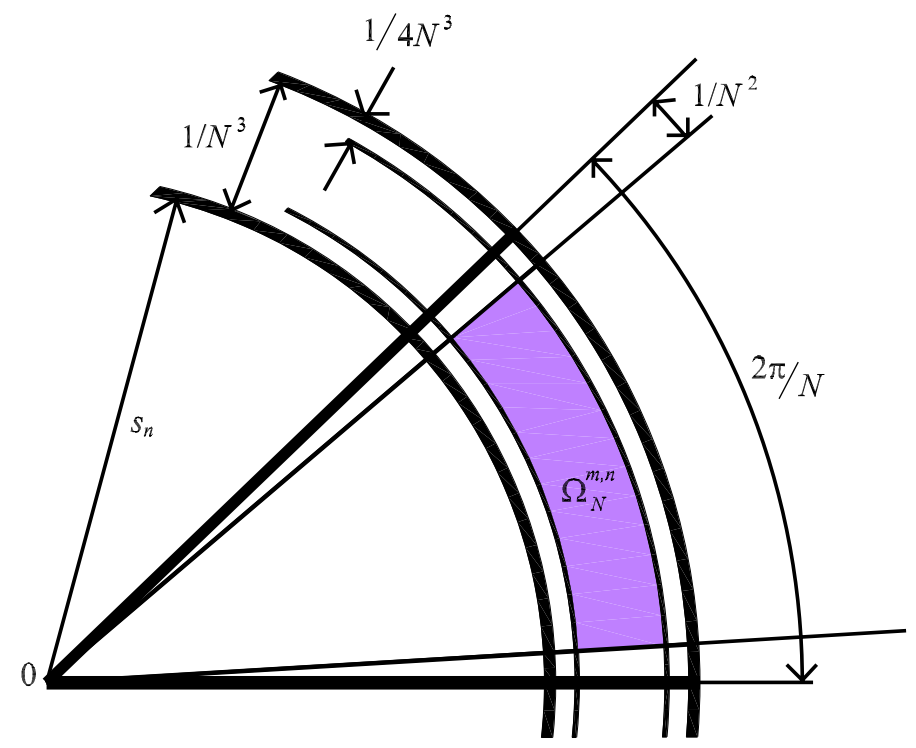

FIGURE 2. The set $\Omega_{N}^{m, n}$.

The shape of the labyrinth formed by the sets $\omega_{i}$, guarantee the following claims if $N$ is large enough: 


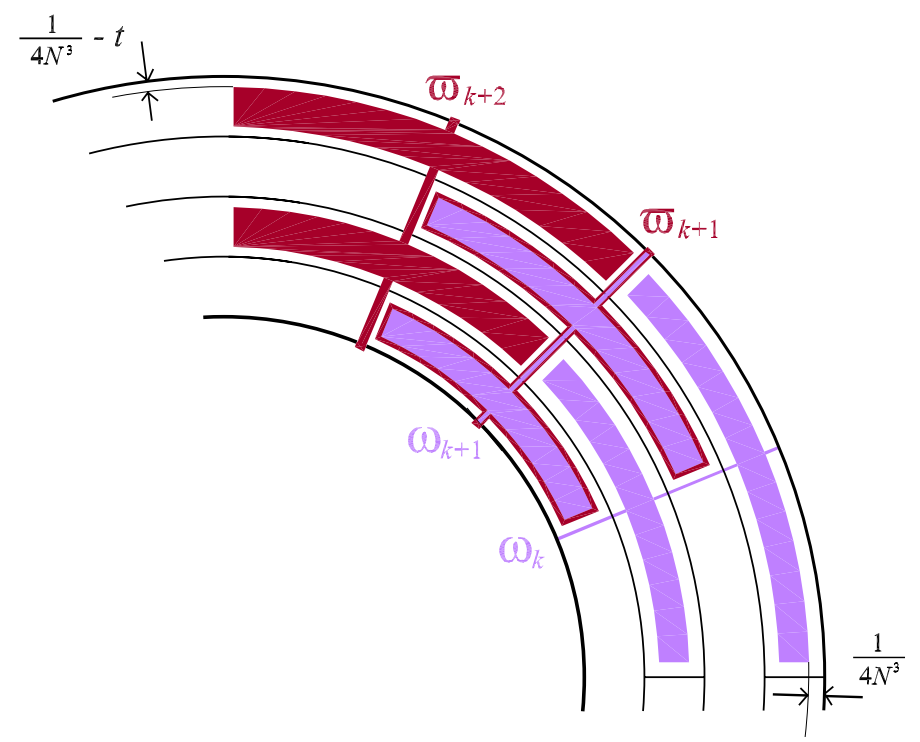

FIGURE 3. The sets $\omega_{k}$ and $\varpi_{k}$.

Claim 2.1. The Euclidean diameter of $\varpi_{i}$ is less than $\frac{\text { const. }}{N}$.

Claim 2.2. If $\lambda^{2}\langle\cdot, \cdot\rangle$ is a conformal metric on $\overline{\mathbb{D}}$ and verifies

$$
\lambda \geq \begin{cases}c & \text { in } \mathbb{D}, \\ c N^{4} & \text { in } \Omega_{N}\end{cases}
$$

for $c \in \mathbb{R}^{+}$, and if $\alpha$ is a curve in $\overline{\mathbb{D}}$ connecting $\mathbb{S}_{1-2 / N}^{1}$ and $\mathbb{S}^{1}$, then

$$
\text { length }(\alpha, \lambda\langle\cdot, \cdot\rangle)>\frac{c \cdot \text { const } \cdot N}{2} \text {. }
$$

Claim 2.2 is a consequence of the fact that a curve $\alpha$, that does not go through the connected components of $\Omega_{N}$, must have a large Euclidean length.

We will deform the initial immersion $X$ by using the López-Ros transformation. So, we will construct a finite sequence of minimal immersions (with boundary); $F_{0}=X, F_{1}, \ldots, F_{2 N}$, in such a way that the last immersion will be the immersion $Y$ that proves the lemma. As all the above immersions are defined on $\overline{\mathbb{D}}$, then the Weierstrass representation of the immersion $F_{i}$ can be written as $\Phi^{i}=\phi^{i}(z) d z$. For our purposes, it is necessary that $F_{i}$ : $\overline{\mathbb{D}} \rightarrow \mathbb{R}^{3}, i \geq 1$, satisfies:

(A. $\left.1_{i}\right)\left\|\phi^{i}(z)-\phi^{i-1}(z)\right\| \leq \frac{1}{N^{2}}, \forall z \in \overline{\mathbb{D}}-\varpi_{i}$

(A. $\left.2_{i}\right)\left\|\phi^{i}(z)\right\| \geq N^{7 / 2}, \forall z \in \omega_{i}$;

(A. $\left.3_{i}\right)\left\|\phi^{i}(z)\right\| \geq \frac{\text { const }}{\sqrt{N}}, \forall z \in \varpi_{i}$;

$\left(\mathrm{A} .4_{i}\right) \operatorname{dist}_{\left(\mathbb{S}^{2}, d s_{0}\right)}\left(G_{i}(z), G_{i-1}(z)\right)<\frac{1}{N^{2}}, \forall z \in \overline{\mathbb{D}}-\varpi_{i}$,

where $G_{i}: \overline{\mathbb{D}} \rightarrow \mathbb{S}^{2}$ represents the spherical Gauss map of the immersion $F_{i}$ and $d s_{0}$ means the Euclidean metric of $\mathbb{S}^{2}$

(A. $\left.5_{i}\right)$ There exists an orthonormal basis of $\mathbb{R}^{3}, S_{i}=\left\{e_{1}^{i}, e_{2}^{i}, e_{3}^{i}\right\}$, so that:

(A.5.1 $\left.{ }_{i}\right)$ If $z \in \varpi_{i}$ and $\|X(z)-\gamma(z /|z|)\| \geq \frac{1}{\sqrt{N}}$, then $\left\|(X(z)-\gamma(z /|z|))_{\left(*, S_{i}\right)}\right\|<\frac{\text { const }}{\sqrt{N}}$;

$\left.(\text { A.5.2 })_{i}\right)\left(F_{i}(z)\right)_{\left(3, S_{i}\right)}=\left(F_{i-1}(z)\right)_{\left(3, S_{i}\right)}, \forall z \in \overline{\mathbb{D}}$;

(A. $\left.6_{i}\right)\left\|F_{i}(z)-F_{i-1}(z)\right\| \leq \frac{\text { const }}{N^{2}}, \forall z \in \overline{\mathbb{D}}-\varpi_{i}$. 
In order to obtain the sequence $F_{0}, \ldots, F_{2 N}$ we follow an inductive method. Assume we have constructed $F_{0}, \ldots, F_{i-1}$ verifying the corresponding Properties $\left(\mathrm{A} .1_{k}\right), \ldots,\left(\mathrm{A} .7_{k}\right)$, for $k=1, \ldots, i-1$. We define $F_{i}$ as follows.

First, observe that (for a large enough $\mathrm{N}$ ) one has:

(B.1) There are positive constants so that const ${ }_{1} \leq\left\|\phi^{i-1}(z)\right\| \leq$ const $_{2}$, for all $z \in \overline{\mathbb{D}}-\cup_{k=1}^{i-1} \varpi_{k}$;

To obtain this property, it suffices to apply $\left(\mathrm{A}_{j}\right)$ for $j=1, \ldots, i-1$.

(B.2) The diameter in $\mathbb{R}^{3}$ of $F_{i-1}\left(\varpi_{i}\right)$ is less than $\frac{1}{\sqrt{N}}$.

This is a consequence of (B.1), Claim 3.1, and (4).

(B.3) The diameter in $\mathbb{S}^{2}$ of $G_{i-1}\left(\varpi_{i}\right)$ is less than $\frac{1}{\sqrt{N}}$. In particular, the set $G_{i-1}\left(\varpi_{i}\right)$ can be included in a cone Cone $\left(g, \frac{1}{\sqrt{N}}\right)$, for a suitable $g \in G_{i-1}\left(\varpi_{i}\right)$.

From Claim 2.1, the diameter of $G_{0}\left(\varpi_{i}\right)$ is bounded. Then (B.3) holds after successive applications of $\left(\mathrm{A} .4_{j}\right), j=1, \ldots, i-1$.

(B.4) There exists an orthogonal frame $S_{i}=\left\{e_{1}, e_{2}, e_{3}\right\}$ in $\mathbb{R}^{3}$, where:

(B.4.1) If $z \in \varpi_{i}$ and $\|X(z)-\gamma(z /|z|)\| \geq \frac{1}{\sqrt{N}}$, then $\angle\left(e_{3}, X(z)-\gamma(z /|z|)\right) \leq \frac{\text { const }}{\sqrt{N}}$;

(B.4.2) $\angle\left( \pm e_{3}, G_{i-1}(z)\right) \geq \frac{\text { const }}{\sqrt{N}}$ for all $z \in \varpi_{i}$.

The proof of (B.4) is slightly more complicated. Let $C$ be the cone Cone $\left(g, \frac{2}{\sqrt{N}}\right)$ where $g$ is given by Property (B.3). Let $\mathcal{N}$ define:

$$
\mathcal{N}:=\left\{\frac{X(z)-\gamma(z /|z|)}{\|X(z)-\gamma(z /|z|)\|} \quad: z \in \varpi_{i} \text { and }\|X(z)-\gamma(z /|z|)\| \geq \frac{1}{\sqrt{N}}\right\} .
$$

To obtain (B.4.2) it suffices to take $e_{3}$ in $\mathbb{S}^{2}-H$, where $H=C \bigcup(-C)$. On the other hand, in order to verify (B.4.1), the vector $e_{3}$ must be chosen as follows:

- If $\left(\mathbb{S}^{2}-H\right) \cap \mathcal{N} \neq \emptyset$, then we take $e_{3} \in\left(\mathbb{S}^{2}-H\right) \cap \mathcal{N}$;

- If $\left(\mathbb{S}^{2}-H\right) \cap \mathcal{N}=\emptyset$, then we take $e_{3} \in \mathbb{S}^{2}-H$ satisfying $\angle\left(e_{3}, q^{\prime}\right)<\frac{2}{\sqrt{N}}$ for some $q^{\prime} \in \mathcal{N}$.

It is straightforward to check that this choice of $e_{3}$ guarantees (B.4).

At this point we are able to construct the $i$-th element of our sequence $F_{i}$. Let $\left(g^{i-1}, \phi_{3}^{i-1}\right)$ be the Weierstrass data of $F_{i-1}$ in the orthonormal basis $S_{i}$. Applying Runge's theorem, we can construct a family of holomorphic functions, $h_{\alpha}: \mathbb{C} \rightarrow \mathbb{C}^{*}$ verifying:

(a) $\left|h_{\alpha}(z)-1\right|<\frac{1}{\alpha}$, for all $z \in \overline{\mathbb{D}}-\varpi_{i}$;

(b) $\left|h_{\alpha}(z)-\alpha\right|<\frac{1}{\alpha}$, for all $z \in \omega_{i}$;

where $\alpha \in \mathbb{R}_{+}$.

Using $h_{\alpha}$ as a López-Ros function, we define this new Weierstrass data:

$$
g^{i}:=\frac{g^{i-1}}{h_{\alpha}}, \quad \Phi_{3}^{i}:=\Phi_{3}^{i-1},
$$

and the associated conformal minimal immersion:

$$
F_{i}(z):=\frac{1}{2} \operatorname{Re} \int^{z}\left(\frac{1}{g^{i}}-g^{i}, \mathrm{i}\left(\frac{1}{g^{i}}+g^{i}\right), 2\right) \Phi_{3}^{i} .
$$

Now, we have to check that $F_{i}$ satisfies Properties (A. $\left.1_{i}\right), \ldots,\left(\mathrm{A} .6_{i}\right)$.

Since $h_{\alpha} \rightarrow 1$, uniformly on $\overline{\mathbb{D}}-\varpi_{i}$, and $h_{\alpha} \rightarrow \infty$, uniformly on $\omega_{i}$, as $\alpha \rightarrow \infty$, then $\left.(\mathbf{A . 1})_{i}\right),\left(\mathbf{A . 2}_{i}\right),\left(\mathbf{A . 4}_{i}\right)$ and $\left(\mathbf{A . 6}_{i}\right)$ trivially hold if $\alpha$ is large enough in terms of $N$.

In order to check (A.3 ${ }_{i}$ ) we have to use (B.4.2). This property gives us:

$$
\frac{\sin \left(\frac{\text { const }}{\sqrt{N}}\right)}{1+\cos \left(\frac{\text { const }}{\sqrt{N}}\right)} \leq\left|g^{i-1}\right| \leq \frac{\sin \left(\frac{\text { const }}{\sqrt{N}}\right)}{1-\cos \left(\frac{\text { const }}{\sqrt{N}}\right)} \quad \text { in } \varpi_{i},
$$

and so, taking (B.1) into account one has (if $N$ is large enough):

$$
\left\|\phi^{i}\right\| \geq\left|\phi_{3}^{i}\right|=\left|\phi_{3}^{i-1}\right|=\sqrt{2}\left\|\phi^{i-1}\right\| \frac{\left|g^{i-1}\right|}{1+\left|g^{i-1}\right|^{2}} \geq \mathrm{const} \cdot \sin \left(\frac{\text { const }}{\sqrt{N}}\right) \geq \frac{\text { const }}{\sqrt{N}} \quad \text { in } \varpi_{i} .
$$

Using (B.4.1), we get (A.5.1 $i$ ). Finally, to obtain (A.5.2 ${ }_{i}$ ), we only use that $\phi_{3}^{i-1}=\phi_{3}^{i}$ in the frame $S_{i}$. 
Hence, we have constructed $F_{0}, F_{1}, \ldots, F_{2 N}$ verifying claims $\left(\right.$ A. $\left.1_{i}\right), \ldots,\left(\right.$ A. $\left.6_{i}\right)$, for $i=1, \ldots, 2 N$. The following proposition stands all the properties of $F_{2 N}$ we will need.

Proposition 1. If $N$ is sufficiently large, then we have:

(I) $\operatorname{dist}_{\left(\overline{\mathbb{D}}, F_{2 N}\right)}\left(\mathbb{S}^{1}, \mathbb{S}^{1}{ }_{1-2 / N}\right)>2 s$;

(II) $\left\|F_{2 N}(z)-X(z)\right\|<$ const $/ N$, for all $z \in \overline{\mathbb{D}}-\bigcup_{i=1}^{2 N} \varpi_{i}$;

(III) There exists a Jordan curve $C$ in $\mathbb{C}$ such that:

(III. a) $\overline{\mathbb{D}_{1-2 / N}} \subset \operatorname{Int} C \subset \overline{\operatorname{Int} C} \subset \mathbb{D}$;

(III. b) $\left.s<\operatorname{dist} \frac{}{(\operatorname{Int} C}, F_{2 N}\right)\left(z, \mathbb{S}^{1}{ }_{1-2 / N}\right)<2 s$, for all $z$ in $C$;

(III. c) $\operatorname{dist}_{\left(\overline{\operatorname{Int} C}, F_{2 N}\right)}(0, C)>\rho+s$;

(III. d) $F_{2 N}(C)$ is a Jordan curve in $\mathbb{R}^{3}$;

(III. e) For any $z \in C$ one has $\left\|F_{2 N}(z)-\gamma(z /|z|)\right\|<R^{\prime}$, where $R^{\prime}=\sqrt{(2 s)^{2}+r^{2}}+\epsilon / 2$.

Proof. Item (I) is an standard consequence of Claim 2.2, making use of (A. $\left.1_{i}\right),\left(\mathrm{A} .2_{i}\right)$ and (A. $\left.3_{i}\right), i=1, \ldots, 2 N$. Similarly, a successive application of (A. $\left.6_{i}\right)$ implies item (II).

The demonstration of item (III) is a bit more delicate. First we need to construct the Jordan curve $C$ in (III). To do this, we consider the set:

$$
\mathcal{A}=\left\{z \in \overline{\mathbb{D}}-\mathbb{D}_{1-2 / N}: s<\operatorname{dist}_{\left(\overline{\mathbb{D}}, F_{2 N}\right)}\left(z, \mathbb{S}_{1-2 / N}^{1}\right)<2 s\right\}
$$

Note that $\mathcal{A}$ is nonempty and that $\mathbb{S}^{1}$ and $\mathbb{S}^{1}{ }_{1-2 / N}$ are in different connected components of $\mathbb{C}-\mathcal{A}$. Then, the existence of $C$ satisfying items (III.a) and (III.b) is obvious. Furthermore, it is also clear that, up to infinitesimal variations of the curve, we can always guarantee that $F_{2 N}(C)$ has no self-intersections (item (III.d).) Item (III.c) is a consequence of $\left(\right.$ A. $\left.1_{i}\right), i=1, \ldots, 2 N$, item (III.b) and the fact that dist $(\overline{\mathbb{D}}, X)\left(0, \mathbb{S}_{1-2 / N}^{1}\right)>\rho$. Now, we are going to check that $F_{2 N}$ verifies item (III.e). Consider $\eta \in C$. We will assume that $\left\|F_{2 N}(\eta)-\gamma(\eta /|\eta|)\right\|>0$, otherwise we have nothing to prove. Hence, we distinguish two cases:

Case 1. The point $\eta$ belongs to $\mathbb{D}-\bigcup_{i=1}^{2 N} \varpi_{i}$.

In this case we know that $\left\|F_{2 N}(\eta)-X(\eta)\right\| \leq \frac{\text { const }}{N}$. Since $\|X(\eta)-\gamma(\eta /|\eta|)\|<r$, then we can choose $N$ large enough so that $\left\|F_{2 N}(\eta)-\gamma(\eta /|\eta|)\right\|<R^{\prime}$.

Case 2. There exists $i \in\{1, \ldots, 2 N\}$ such that $\eta \in \varpi_{i}$.

Consider now a curve $\beta:[0,1] \rightarrow \operatorname{Int} C$ so that $\beta(0) \in \mathbb{S}_{1-\epsilon}^{1}, \beta(1)=\eta$ and length $\left(\beta, F_{2 N}\right) \leq 2 s$. Note that the existence of such a curve is guaranteed by (III.b). Let us define $\bar{t}=\sup \left\{t \in[0,1]: \beta(t) \in \partial \varpi_{i}\right\}$ and $\bar{\eta}=\beta(\bar{t})$. It is important to note that $\bar{t}$ exists because $\varpi_{i} \subset \overline{\mathbb{D}}-\mathbb{D}_{1-2 / N}$.

For our purposes, we need to prove the following inequality first:

$$
\left\|F_{i}(\bar{\eta})-F_{i}(\eta)\right\| \leq \frac{\mathrm{const}}{N}+2 s .
$$

Indeed, by using Properties (A. $6_{k}$ ), for $k=1, \ldots, 2 N$, we obtain:

$$
\begin{array}{r}
\left\|F_{i}(\bar{\eta})-F_{i}(\eta)\right\| \leq\left\|F_{i}(\bar{\eta})-F_{2 N}(\bar{\eta})\right\|+\left\|F_{2 N}(\bar{\eta})-F_{2 N}(\eta)\right\|+\left\|F_{2 N}(\eta)-F_{i}(\eta)\right\| \leq \\
\frac{\text { const }}{N}+\operatorname{length}\left(\beta, F_{2 N}\right)+\frac{\text { const }}{N} \leq \frac{\text { const }}{N}+2 s .
\end{array}
$$

Similarly, if we apply Properties (A. $6_{k}$ ), for $k=i+1, \ldots, 2 N$, then we have:

$$
\left\|F_{2 N}(\eta)-\gamma(\eta /|\eta|)\right\| \leq\left\|F_{i}(\eta)-\gamma(\eta /|\eta|)\right\|+\frac{\text { const }}{N} .
$$

We again distinguish two cases: 
Case 2.1. If $\|X(\eta)-\gamma(\eta /|\eta|)\| \leq 1 / \sqrt{N}$, then one has:

$$
\begin{aligned}
& \left\|F_{i}(\eta)-\gamma(\eta /|\eta|)\right\| \leq\left\|F_{i}(\eta)-F_{i}(\bar{\eta})\right\|+\left\|F_{i}(\bar{\eta})-F_{i-1}(\bar{\eta})\right\|+ \\
& \left\|F_{i-1}(\bar{\eta})-F_{i-1}(\eta)\right\|+\left\|F_{i-1}(\eta)-X(\eta)\right\|+\|X(\eta)-\gamma(\eta /|\eta|)\| \leq \\
& \frac{\text { const }}{N}+2 s+\frac{\text { const }}{N^{2}}+\frac{\text { const }}{\sqrt{N}}+\frac{\text { const }}{N}+\frac{1}{\sqrt{N}} .
\end{aligned}
$$

This inequality and (8) finished the proof of (III.e) for a large enough $N$.

Case 2.2. If $\|X(\eta)-\gamma(\eta /|\eta|)\|>1 / \sqrt{N}$, then we use (A.5.2 $2_{i}$ ) to get a bound for the third coordinate of $F_{i}(\eta)-\gamma(\eta /|\eta|)$ in the orthonormal basis $S_{i}$. We proceed as follows:

(9) $\left|\left(F_{i}(\eta)-\gamma(\eta /|\eta|)\right)_{\left(3, S_{i}\right)}\right|=\left|\left(F_{i-1}(\eta)-\gamma(\eta /|\eta|)\right)_{\left(3, S_{i}\right)}\right| \leq$

$$
\left|\left(F_{i-1}(\eta)-X(\eta)\right)_{\left(3, S_{i}\right)}\right|+\left|(X(\eta)-\gamma(\eta /|\eta|))_{\left(3, S_{i}\right)}\right| \leq \frac{\text { const }}{N}+r
$$

On the other hand, we can apply Property $\left(\mathrm{A} .5 .1_{i}\right)$ to find an upper bound for the first two coordinates of $F_{i}(\eta)-$ $\gamma(\eta /|\eta|)$

$$
\begin{aligned}
& \left\|\left(F_{i}(\eta)-\gamma(\eta /|\eta|)\right)_{\left(*, S_{i}\right)}\right\| \leq\left\|\left(F_{i}(\eta)-F_{i}(\bar{\eta})\right)_{\left(*, S_{i}\right)}\right\|+\left\|\left(F_{i}(\bar{\eta})-F_{i-1}(\bar{\eta})\right)_{\left(*, S_{i}\right)}\right\|+ \\
& \left\|\left(F_{i-1}(\bar{\eta})-F_{i-1}(\eta)\right)_{\left(*, S_{i}\right)}\right\|+\left\|\left(F_{i-1}(\eta)-X(\eta)\right)_{\left(*, S_{i}\right)}\right\|+\left\|(X(\eta)-\gamma(\eta /|\eta|))_{\left(*, S_{i}\right)}\right\| \leq \\
& \frac{\text { const }}{N}+2 s+\frac{\text { const }}{N^{2}}+\frac{\text { const }}{N}+\frac{1}{\sqrt{N}}+\frac{\text { const }}{\sqrt{N}} \leq 2 s+\frac{\text { const }}{\sqrt{N}} .
\end{aligned}
$$

By Pythagoras' theorem and taking into account (9) and (10), we easily infer that:

$$
\left\|F_{i}(\eta)-\gamma(\eta /|\eta|)\right\|<\sqrt{\left(2 s+\frac{\text { const }}{\sqrt{N}}\right)^{2}+\left(r+\frac{\text { const }}{N}\right)^{2}} .
$$

Using this upper bound, inequality (8) becomes:

$$
\left\|F_{2 N}(\eta)-\gamma(\eta /|\eta|)\right\| \leq \sqrt{\left(2 s+\frac{\text { const }}{\sqrt{N}}\right)^{2}+\left(r+\frac{\text { const }}{N}\right)^{2}}+\frac{\text { const }}{N} .
$$

So, for a large enough $N$, it is clear that $\left\|F_{2 N}(\eta)-\gamma(\eta /|\eta|)\right\|<R^{\prime}$ (recall $R^{\prime}=\sqrt{r^{2}+(2 s)^{2}}+\epsilon / 2$ ) for all $\eta \in C$. This completes the proof of item (III.e).

Obviously, the immersion $F_{2 N}: \overline{\operatorname{Int} C} \rightarrow \mathbb{R}^{3}$ is almost the immersion $\widetilde{X}$ we are looking for. Proposition 1 says to us that $F_{2 N}$ is a good candidate to verify the theses of the lemma, but it is not defined on the unit disk $\mathbb{D}$.

In order to solve this problem, we consider a holomorphic diffeomorphism $\Sigma: \overline{\mathbb{D}} \rightarrow \overline{\operatorname{Int} C}$, fulfilling:

- $\Sigma(0)=0$,

- $\Sigma^{\prime}(0) \in \mathbb{R}^{+}$.

The uniform continuity of $\gamma$ implies the existence of $\delta>0$ such that:

$$
\|\gamma(x)-\gamma(y)\|<\frac{\epsilon}{2}, \quad \text { whenever } \quad|x-y|<\delta .
$$

Moreover, a convergence theorem by Carathéodory (see $[4, \S 5$, Theorem 1$]$ ) says to us that $\Sigma$ converges to Id $\mathbb{D}$ uniformly on compact sets of $\mathbb{D}$, as $N$ goes to $\infty$. So, if $N$ is large enough, we can guarantee that:

$$
\left|\frac{\Sigma(z)}{|\Sigma(z)|}-z\right|<\delta, \quad \forall z \in \mathbb{S}^{1}
$$

At this point we define $\tilde{X}=F_{2 N} \circ \Sigma$. Let us check that $\tilde{X}$ satisfies all the items in the statement of the lemma.

Items (a) and (b) are direct consequences of (III.c) and (III.d) in Proposition 1, respectively.

In order to check item (c), take a point $z$ in $\mathbb{S}^{1}$. Then, we can use (11), (12) and item (III.e) in Proposition 1 to deduce:

$$
\|\tilde{X}(z)-\gamma(z)\| \leq\left\|F_{2 N}(\Sigma(z))-\gamma\left(\frac{\Sigma(z)}{|\Sigma(z)|}\right)\right\|+\left\|\gamma\left(\frac{\Sigma(z)}{|\Sigma(z)|}\right)-\gamma(z)\right\|<R^{\prime}+\frac{\epsilon}{2}=R .
$$


This completes the proof of Lemma 3.

Lemma 4 (Main Lemma). Let $X: \overline{\mathbb{D}} \rightarrow \mathbb{R}^{3}$ be a conformal minimal immersion, so that $X\left(\mathbb{S}^{1}\right)$ is a Jordan curve. Then, for any $\mu>0$ there exists a conformal minimal immersion $Y: \overline{\mathbb{D}} \rightarrow \mathbb{R}^{3}$ satisfying:

(i) $\|Y-X\|<\mu$, in $\mathbb{D}$;

(ii) $\operatorname{dist}_{Y}\left(0, \mathbb{S}^{1}\right)>\operatorname{dist}_{X}\left(0, \mathbb{S}^{1}\right)+1$;

(iii) length $\left(Y\left(\mathbb{S}^{1}\right)\right)>$ length $\left(X\left(\mathbb{S}^{1}\right)\right)$;

(iv) $Y\left(\mathbb{S}^{1}\right)$ is a Jordan curve.

Proof. Let $c_{0}>0, r_{1}>0$ and $\rho_{1}>0$ to be specified later, and define

$$
r_{n}=\sqrt{r_{n-1}^{2}+\left(\frac{2 c_{0}}{n}\right)^{2}}+\frac{c_{0}}{n^{2}},
$$

and $\rho_{n}=\rho_{1}+\sum_{i=2}^{n} c_{0} / i, n \geq 2$. The constants $r_{1}$ and $c_{0}$ are chosen in such a way that:

$$
\lim _{n \rightarrow \infty} r_{n}<\mu
$$

Our strategy consists of using Lemma 3 to define a sequence:

$$
\chi_{n}=\left(X_{n}: \overline{\mathbb{D}} \rightarrow \mathbb{R}^{3}, \varepsilon_{n}\right),
$$

where $\left\{X_{n}\right\}_{n \in \mathbb{N}}$ is a sequence of conformal minimal immersions and $\left\{\varepsilon_{n}\right\}$ is a decreasing sequence of non vanishing terms satisfying :
$\left(\mathrm{A}_{n}\right) \varepsilon_{n}<c_{0} / n^{2}$,
$\left(\mathrm{B}_{n}\right) \rho_{n}<\operatorname{dist}_{\left(X_{n}, \overline{\mathbb{D}}\right)}\left(0, \mathbb{S}^{1}\right)$,
$\left(\mathrm{C}_{n}\right)\left\|X_{n}(z)-X(z)\right\|<r_{n}$, for all $z$ in $\mathbb{S}^{1}$,
$\left(\mathrm{D}_{n}\right) X_{n}\left(\mathbb{S}^{1}\right)$ is a Jordan curve.

The choice of the first element of the sequence is not difficult. First, we take $X_{1}=X$. Finally, we choose $\rho_{1}$ and $\varepsilon_{1}$ satisfying:

$$
\rho_{1}<\operatorname{dist}_{\left(X_{1}, \overline{\mathbb{D}}\right)}\left(0, \mathbb{S}^{1}\right) \quad \text { and } \quad \varepsilon_{1}<\min \left\{c_{0}, r_{1}\right\} .
$$

Suppose that we have defined $\chi_{1}, \ldots, \chi_{n}$. Then, we will construct the $(n+1)$-th term in the following way.

Take a real number $\varepsilon_{n+1}<\min \left\{\varepsilon_{n}, \frac{c_{0}}{(n+1)^{2}}\right\}$. Then we apply Lemma 3 to the data:

$$
X=X_{n}, \Gamma=X\left(\mathbb{S}^{1}\right), \gamma(z)=X(z), r=r_{n}, s=\frac{c_{0}}{n+1}, \epsilon=\varepsilon_{n+1} .
$$

So, we get a new minimal immersion $X_{n+1}$ that satisfies the desired properties $\left(\mathrm{A}_{n+1}\right), \ldots,\left(\mathrm{D}_{n+1}\right)$.

As the sequence $\left\{\rho_{n}\right\}_{n \in \mathbb{N}}$ diverges, then it is clear that there exists $n_{0} \in \mathbb{N}$ such that $\rho_{n}>\operatorname{dist}(\overline{\mathbb{D}}, X)\left(0, \mathbb{S}^{1}\right)+1$, for all $n \geq n_{0}$.

On the other hand, using Bishop's comparison theorems (see $[1, \S 3.4])$, we have that the area $\mathrm{A}\left(X_{n}(\overline{\mathbb{D}})\right) \geq \pi \rho_{n}^{2}$. In particular this area diverges. By the isoperimetric inequality $\left\{\operatorname{length}\left(X_{n}\left(\mathbb{S}^{1}\right)\right)\right\}_{n \in \mathbb{N}}$ diverges too. Hence, there exists $n_{1} \in \mathbb{N}$ such that length $\left(X_{n}\left(\mathbb{S}^{1}\right)\right)>\operatorname{length}\left(X\left(\mathbb{S}^{1}\right)\right)$, for all $n \geq n_{1}$.

Label $m:=\max \left\{n_{0}, n_{1}\right\}$ and $Y:=X_{m}$. From Property $\left(\mathrm{C}_{m}\right)$, we have:

$$
\|Y(z)-X(z)\| \leq r_{m}<\mu, \quad \forall z \in \mathbb{S}^{1} .
$$

The Maximum Principle for harmonic maps implies that the last inequality occurs for any $z$ in $\overline{\mathbb{D}}$. This completes the proof of the lemma. 


\section{THE MAIN THEOREM}

The main result of this paper is now a trivial consequence of Lemma 4.

Theorem 1. There exists a complete, conformal, minimal immersion $f: \mathbb{D} \longrightarrow \mathbb{R}^{3}$ so that it admits a continuous extension to the closed disk, $F: \overline{\mathbb{D}} \longrightarrow \mathbb{R}^{3}$. The boundary curve, $F\left(\mathbb{S}^{1}\right)$, is a (non-rectifiable) Jordan curve with Hausdorff dimension 1.

Moreover, for any $\Gamma$ Jordan curve in $\mathbb{R}^{3}$ and any $\xi>0$, we can find a minimal immersion satisfying the above conditions and such that:

$$
\delta^{H}\left(\Gamma, F\left(\mathbb{S}^{1}\right)\right)<\xi
$$

Proof. Let $\Gamma$ be a Jordan curve in Euclidean space and fix $\xi$ a positive constant. It is well known that there exists an analytic Jordan curve $\Gamma_{1}$ such that $\delta^{H}\left(\Gamma_{1}, \Gamma\right)<\xi / 2$. Let $M_{1}$ be a minimal disk spanned by $\Gamma_{1}$ and $F_{1}: \overline{\mathbb{D}} \rightarrow M_{1}$ a conformal parameterization of $M_{1}$.

Now, we will construct the immersion $f$ as a limit of a sequence of compact minimal disks with boundary, $F_{n}: \overline{\mathbb{D}} \rightarrow \mathbb{R}^{3}$, satisfying:

$\left(1_{\mathrm{n}}\right) F_{n}\left(\mathbb{S}^{1}\right)$ is a Jordan curve;

$\left(2_{\mathrm{n}}\right) \operatorname{dist}_{\left(\mathbb{D}, F_{n}\right)}\left(0, \mathbb{S}^{1}\right)>n+\operatorname{dist}_{\left(\mathbb{D}, F_{1}\right)}\left(0, \mathbb{S}^{1}\right)$

$\left(3_{\mathrm{n}}\right)\left\|F_{n}(z)-F_{n-1}(z)\right\|<\xi_{n}, \forall z \in \overline{\mathbb{D}}$, where:

- $\xi_{n}<\varphi\left(\left(F_{n-1}\right)_{\mid \mathbb{S}^{1}}, n\right)$;

- $\sum_{n=1}^{\infty} \xi_{n}<\xi / 2$;

$\left(4_{\mathrm{n}}\right) \lambda_{F_{n}}(z) \geq \alpha_{n} \lambda_{F_{n-1}}(z), \forall z \in \overline{\mathbb{D}}_{1-1 / n}$, where $0<\alpha_{i}<1$ and $\left\{\prod_{i=1}^{n} \alpha_{i}\right\}_{n}$ converges to $1 / 2 ;$

$\left(5_{\mathrm{n}}\right)$ length $\left(F_{n}\left(\mathbb{S}^{1}\right)\right)>\operatorname{length}\left(F_{n-1}\left(\mathbb{S}^{1}\right)\right)$.

The above sequence is defined in a recursive way. The first element of the sequence is the aforementioned immersion $F_{1}$. The number $\xi_{1}$ is chosen as follows. Consider a convergent series of positive terms $a_{n}>0, n \in \mathbb{N}$, satisfying $\sum_{n=1}^{\infty} a_{n}=\xi / 2$. Then we take $\xi_{1}$ verifying $0<\xi_{1}<a_{1}$.

Assume we have constructed $F_{1}, \ldots, F_{n}$, satisfying the corresponding properties. We are going to define $F_{n+1}$. In order to do this, we consider a sequence $\left\{\mu_{m}\right\}_{m \in \mathbb{N}}$ so that

$$
\mu_{m}<\min \left\{a_{n+1}, \varphi\left(\left(F_{n}\right)_{\mid \mathbb{S}^{1}}, n+1\right)\right\}, \quad \forall m \in \mathbb{N} .
$$

If we apply Lemma 4 to the following data:

$$
\begin{aligned}
& \text { - } X=F_{n}: \overline{\mathbb{D}} \rightarrow \mathbb{R}^{3}, \\
& \text { - } \mu=\mu_{m}, m \in \mathbb{N},
\end{aligned}
$$

then we get a sequence of minimal immersions with boundary $Y_{m}: \overline{\mathbb{D}} \rightarrow \mathbb{R}^{3}$ that converges to $F_{n}$, as $m \rightarrow \infty$. In particular, $\lambda_{Y_{m}}$ converges uniformly to $\lambda_{F_{n}}$ on $\overline{\mathbb{D}}_{1-1 /(n+1)}$. Therefore, there is a $m_{0} \in \mathbb{N}$ such that, for any $m \geq m_{0}$, one has:

$$
\lambda_{Y_{m}}(z) \geq \alpha_{n+1} \lambda_{F_{n}}(z), \quad \forall z \in \overline{\mathbb{D}}_{1-1 /(n+1)} .
$$

Thus, we define $F_{n+1}:=Y_{m_{0}}$ and $\xi_{n+1}=\mu_{m_{0}}$. It is clear that they satisfy Properties $\left(1_{n+1}\right), \ldots,\left(5_{n+1}\right)$.

Reasoning as in the proof of Lemma 4 (page 10), we deduce that the sequence $\left\{\operatorname{length}\left(F_{n}\left(\mathbb{S}^{1}\right)\right)\right\}_{n \in \mathbb{N}}$ diverges. Moreover, Properties $\left(3_{n}\right)$ and $\left(5_{n}\right), n \in \mathbb{N}$, tell us that the assumptions of Lemma 2 are satisfied. Then the sequence $\left\{F_{n}\right\}_{n \in \mathbb{N}}$ converges uniformly to a continuous map $F: \overline{\mathbb{D}} \rightarrow \mathbb{R}^{3}, F_{\mid \mathbb{S}^{1}}: \mathbb{S}^{1} \rightarrow \mathbb{R}^{3}$ is one-to-one, and $F\left(\mathbb{S}^{1}\right)$ is a Jordan curve of Hausdorff dimension 1.

Furthermore, making use of Harnack's theorem, we know that $f:=F_{\mid \mathbb{D}}: \mathbb{D} \rightarrow \mathbb{R}^{3}$ is a harmonic map. Let us see that $f$ is an immersion. Take $z \in \mathbb{D}$, there exists $k \in \mathbb{N}$ so that $z \in \mathbb{D}_{1-1 / n}, \forall n \geq k$. Then, for any $n \geq k$ we 
use Properties $\left(4_{n}\right)$ to obtain:

$$
\lambda_{F_{n}}(z) \geq \alpha_{n} \lambda_{F_{n-1}}(z) \geq \cdots \geq \prod_{i=k}^{n} \alpha_{i} \lambda_{F_{k-1}}(z) \geq \frac{1}{2} \lambda_{F_{k-1}}(z)>0 .
$$

If we take limit in the above inequality as $n \rightarrow \infty$, then we get $\lambda_{f}(z)>0$.

The completeness of immersion $f$ is a trivial consequence of Properties $\left(2_{n}\right), n \in \mathbb{N}$.

Finally, notice that for any $z \in \mathbb{S}^{1}$ one has:

$$
\left\|F(z)-F_{1}(z)\right\| \leq \sum_{n=1}^{\infty}\left\|F_{n+1}(z)-F_{n}(z)\right\|<\sum_{n=1}^{\infty} \xi_{n}<\xi / 2 .
$$

In particular $\delta^{H}\left(F\left(\mathbb{S}^{1}\right), F_{1}\left(\mathbb{S}^{1}\right)\right)<\xi / 2$. The choice that we made of $F_{1}\left(\mathbb{S}^{1}\right)$ gives us that $\delta^{H}\left(\Gamma, F\left(\mathbb{S}^{1}\right)\right)<\xi$. This concludes the proof.

Remark 2. From the arguments used in the proof of Lemmas 3 and 4 , it is possible to deduce that $F\left(\mathbb{S}^{1}\right)$ is nonrectifiable. However, there are extra reasons to deduce this fact. If a minimal surface spans a rectifiable Jordan curve, then by the isoperimetric inequality for minimal surfaces it should have a finite area, but this is absurd (to see this contradiction we only have to apply Bishop's comparison theorem.)

\section{REFERENCES}

[1] I. Chavel, Riemannian geometry-a modern introduction. Cambridge Tracts in Mathematics, 108. Cambridge University Press, Cambridge, 1993.

[2] T. H. Colding and W. P. Minicozzi II, The Calabi-Yau conjectures for embedded surfaces. Preprint math.DG/0404197 (2004).

[3] U. Dierkes, S. Hildebrandt, A. Küster and O. Wohlrab Minimal surfaces. I. Grundlehren der Mathematischen Wissenschaften, vol. 295. Springer-Verlag, Berlin, 1992.

[4] G. M. Goluzin, Geometric Theory of Functions of a Complex Variable. Translations of Mathematical Monographs, Vol. 46. American Math. Society, Providence, RI, 1969.

[5] F. Martín and S. Morales, Complete proper minimal surfaces in convex bodies of $\mathbb{R}^{3}$, II. The behavior of the limit set. Comment. Math. Helv. (to appear).

[6] F. Morgan, Geometric measure theory. A beginner's guide. Third edition. Academic Press, Inc., San Diego, CA, 2000.

[7] N. Nadirashvili, Hadamard's and Calabi-Yau's conjectures on negatively curved and minimal surfaces. Invent. Math. 126 (1996), 457465.

[8] H. Werner,The existence of surfaces of constant mean curvature with arbitrary Jordan curves as assigned boundary. Proc. Amer. Math. Soc. $11196063-70$.

DEPARTAMENTO DE GEOMETRÍA y TOPOLOGÍA

UNIVERSIDAD DE GRANADA,

18071, GRANADA

SPAIN

CNRS, LATP, CMI

39, RUE JOLIOT-CURIE

13453 MARSEILLE CEDEX 13

FRANCE

E-mail address, F. Martín: f ma r t i n @ugr . es

E-mail address, N. Nadirashvili: ni col as @c mi . uni v-mr s.fr 